\title{
ON THE CONVERGENCE-ABSCISSAS OF THE GENERALIZED FACTORIAL SERIES
}

\author{
CHUJI TANAKA
}

1. Introduction. We consider the generalized factorial series

$$
\begin{array}{r}
F(s)=\sum_{n=1}^{\infty} a_{n}\left[\lambda_{1} \lambda_{2} \cdots \lambda_{n}\right]\left[\left(s+\lambda_{1}\right)\left(s+\lambda_{2}\right) \cdots\left(s+\lambda_{n}\right)\right]^{-1}, \\
s=\sigma+i t, \lambda_{n}=r_{n} e^{i \phi_{n}}(n=1,2, \cdots),
\end{array}
$$

where

$$
\lim _{n \rightarrow \infty} r_{n}=+\infty,\left|\phi_{n}\right| \leqq \phi<\pi / 2 \quad(n=1,2, \cdots) .
$$

In his classical note $[1, \S 6]$, E. Landau has studied (1.1) in the case in which $\sum_{n=1}^{\infty} 1 / r_{n}=+\infty, \phi_{n}=0(n=1,2, \cdots)$. Under additional conditions, he has determined convergence-abscissas of (1.1) in terms of coefficients $a_{n}(n=1,2, \cdots)$. S. Pincherle [2], G. Belardinelli $[3]$, and T. Fort $[4,5]$ have studied (1.1) with complex $\lambda_{n}$ $(n=1,2, \cdots)$ satisfying $(1.2)$ and some other conditions. In this note, without any additional conditions, we shall determine the convergence-abscissas of (1.1) with real $\lambda_{n}(n=1,2, \cdots)$ in terms of coefficients $a_{n}(n=1,2, \cdots)$. In the case in which the $\lambda_{n}$ are complex, the convergence-domains of (1.1) are not generally halfplanes, and so the convergence-abscissas of (1.1) have no meaning.

The main theorems are:

Theorem I. In the case $\phi_{n}=0(n=1,2, \cdots),(1.1)$ has three convergence-abscissas, i.e. a simple convergence-abscissa $\sigma_{\bullet}$, a uniform convergence-abscissa $\sigma_{u}$, and an absolute convergence-abscissa $\sigma_{a}$ such that $\sigma_{\varepsilon}=\sigma_{u} \leqq \sigma_{a}$.

REMARK. (1) In the convergence-problem of (1.1), the sequence of points $-\lambda_{n}(n=1,2, \cdots)$ is excluded from the $s$-plane by small circles with centres at $-\lambda_{n}(n=1,2, \cdots)$ and radii $\epsilon, \epsilon$ being a small positive constant.

(2) The divergence of $\sum_{n=1}^{\infty} 1 / r_{n}$ is not necessary for the validity of Theorem 1.

THEOREM II. If $\sum_{n=1}^{\infty} 1 / r_{n}<+\infty$, the necessary and sufficient condition for (1.1) to be simply (absolutely) convergent at $s=s_{0}$ distinct from $-\lambda_{n}(n=1,2, \cdots)$ is that $\sum_{n=1}^{\infty} a_{n}\left(\sum_{n=1}^{\infty}\left|a_{n}\right|\right)$ converges. If

Received by the editors February 1, 1952. 
furthermore $\phi_{n}=0(n=1,2, \cdots)$, then three possibilities now present themselves:

\begin{tabular}{|c|c|c|c|c|}
\hline Case & $\sum_{n=1}^{\infty}\left|a_{n}\right|$ & $\sum_{n=1}^{\infty} a_{n}$ & $\sigma_{s}=\sigma_{u}$ & $\sigma_{a}$ \\
\hline I & $<+\infty$ & convergent & $=-\infty$ & $=-\infty$ \\
\hline II & $=+\infty$ & convergent & $=-\infty$ & $=+\infty$ \\
\hline III & $=+\infty$ & divergent & $=+\infty$ & $=+\infty$ \\
\hline
\end{tabular}

Theorem III. If $\sum_{n=1}^{\infty} 1 / r_{n}=+\infty, \phi_{n}=0(n=1,2, \cdots)$, then the three convergence-abscissas of (1.1) are determined respectively by

(a) $\quad \sigma_{\iota}=\sigma_{u}=\limsup _{n \rightarrow \infty} 1 / l_{n} \cdot \log \left|\sum_{n=1}^{n} a_{\nu} \exp \left(\phi\left(l_{v}\right)-\phi\left(l_{n}\right)\right)\right|$,

$$
\sigma_{a}=\limsup _{n \rightarrow \infty} 1 / l_{n} \cdot \log \left\{\sum_{n=1}^{n}\left|a_{\nu}\right| \exp \left(\phi\left(l_{n}\right)-\phi\left(l_{n}\right)\right)\right\}
$$

where

$$
l_{n}=\sum_{i=1}^{n} l / r_{i} \quad\left(0<l_{1}<l_{2}<\cdots<l_{n} \rightarrow+\infty\right),
$$

(d) $\phi(x)$ is the positive and differentiable function defined for $x>0$ such that

(i) $\phi(x) \uparrow+\infty, \phi^{\prime}(x) \rightarrow+\infty$ as $x \rightarrow+\infty$.

(ii) for any given $\epsilon>0, \int+\infty \exp (-\epsilon x)\left|\phi^{\prime}(x)\right| d x<+\infty$.

Corollary I (Equiconvergence Theorem) (T. Fort [4, p. 239]). Under the same conditions as in Theorem III, (1.1) has the same abscissa of simple convergence and the same abscissa of absolute convergence as the Dirichlet series

$$
G(s)=\sum_{n=1}^{\infty} a_{n} \exp \left(-l_{n} s\right)
$$

COROLLARY II. Under the same conditions as in Theorem III, we have

(a)

$$
\begin{aligned}
\sigma_{a}=\sigma_{u} & =\limsup _{x \rightarrow \infty} 1 / x \cdot \log \left|\sum_{[x] \leq l_{n}<x} a_{n}\right|, \\
\sigma_{a} & =\limsup _{x \rightarrow \infty} 1 / x \cdot \log \left\{\sum_{[x] \leq l_{n}<x}\left|a_{n}\right|\right\},
\end{aligned}
$$


where $[x]$ denotes the greatest integer contained in $x$.

$$
0 \leqq \sigma_{a}-\sigma_{a} \leqq \limsup _{n \rightarrow \infty} 1 / l_{n} \cdot \log n .
$$

2. Proof of Theorem I. We first prove some necessary lemmas, which are analogues of theorems concerning ordinary factorial series [6, pp. 171-174].

LEMMA I. If (1.1) is simply convergent at $s=s_{0}$, then (1.1) is uniformly convergent in the angular domain $D\left(s_{0}, \vartheta, \phi\right):\left|\arg \left(s-s_{0}\right)\right| \leqq \vartheta$ $<(\pi / 2-\phi)$, where $\vartheta$ is an arbitrary but fixed constant.

As a special case of Lemma I, we have

LEMMA I'. If (1.1) with real $\lambda_{n}(n=1,2, \cdots)$ is simply convergent at $s=s_{0}$, then (1.1) is uniformly convergent in the angular domain $D\left(s_{0}, \vartheta, 0\right):\left|\arg \left(s-s_{0}\right)\right| \leqq \vartheta<\pi / 2$, where $\vartheta$ is an arbitrary but fixed constant.

Under the assumptions that $\lim _{n \rightarrow \infty} \phi_{n}=0$, and $\sum_{n-1}^{\infty} 1 / r_{n}=+\infty$, T. Fort $[4$, p. 237, Theorem IV] has proved that (1.1) converges uniformly in the angular domain $D\left(s_{0}, \vartheta, 0\right)$, provided that it converges simply at $s=s_{0}$. Since we can put $\phi=\epsilon$ in Lemma I, $\epsilon$ being any small positive constant, this theorem is evidently contained in Lemma I.

Proof of Lemma I. We first establish the inequality

$$
\left|s+\lambda_{n}\right|>\left|s_{0}+\lambda_{n}\right|+r \sin (\eta / 2) \quad \text { for } n \geqq n_{1} \text {, }
$$

where

(i) $s \in D\left(s_{0}, \vartheta, \phi\right), r=\left|s-s_{0}\right|, \vartheta=\pi / 2-(\phi+\eta)(\eta>0)$,

(ii) $n_{1}$ is a sufficiently large integer.

In fact, putting $\theta=\arg \left(s-s_{0}\right)-\arg \left(s_{0}+\lambda_{n}\right)$, where $s \in D\left(s_{0}, \vartheta, \phi\right)$, we have easily

so that

$$
\pi / 2+\eta / 2 \leqq \theta<3 \pi / 2-\eta / 2 \quad \text { for } n \geqq n_{1} \text {, }
$$

$$
\begin{aligned}
\left|s+\lambda_{n}\right|^{2} & =r^{2}+\left|s_{0}+\lambda_{n}\right|^{2}-2 r\left|s_{0}+\lambda_{n}\right| \cos \theta \\
& \geqq\left\{\left|s_{0}+\lambda_{n}\right|+r \sin (\eta / 2)\right\}^{2} \quad \text { for } n \geqq n_{1},
\end{aligned}
$$

which proves (2.1). Let us put

$$
\begin{aligned}
b_{n} & =a_{n}\left[\lambda_{1} \cdots \lambda_{n}\right]\left[\left(s_{0}+\lambda_{1}\right)\left(s_{0}+\lambda_{2}\right) \cdots\left(s_{0}+\lambda_{n}\right)\right]^{-1} \\
c_{n}(s) & =\left[\left(s_{0}+\lambda_{1}\right)\left(s_{0}+\lambda_{2}\right) \cdots\left(s_{0}+\lambda_{n}\right)\right]\left[\left(s+\lambda_{1}\right)\left(s+\lambda_{2}\right) \cdots\right. \\
& \left.\left(s+\lambda_{n}\right)\right]^{-1}
\end{aligned}
$$


Equation (2.1) yields

$$
\begin{aligned}
& \left|\left(s_{0}+\lambda_{n}\right) /\left(s+\lambda_{n}\right)\right|<\rho_{n}\left[\rho_{n}+r \sin (\eta / 2)\right]^{-1}, \\
& \left|\left(s-s_{0}\right) /\left(s+\lambda_{n+1}\right)\right|<r\left[\rho_{n+1}+r \sin (\eta / 2)\right]^{-1}, \quad \text { for } n \geqq n_{1},
\end{aligned}
$$

where $s \in D\left(s_{0}, \vartheta, \phi\right), r=\left|s-s_{0}\right|$, and $\rho_{n}=\left|s_{0}+\lambda_{n}\right|$. Hence

$$
\begin{aligned}
\left|c_{n}(s)-c_{n+1}(s)\right| & =\left|c_{n}(s)\left(s-s_{0}\right)\left(s+\lambda_{n+1}\right)^{-1}\right| \\
& <|K(s)| \cdot d_{n} \cdot r\left[\rho_{n+1}+r \sin (\eta / 2)\right]^{-1},
\end{aligned}
$$

where

$K(s)=\left[\left(s_{0}+\lambda_{1}\right)\left(s_{0}+\lambda_{2}\right) \cdots\left(s_{0}+\lambda_{n_{1}-1}\right)\right]\left[\left(s+\lambda_{1}\right)\left(s+\lambda_{2}\right) \cdots\left(s+\lambda_{n_{1}-1}\right)\right]^{-1}$, and

$$
d_{n}=\prod_{i=n_{1}}^{n} \rho_{n}\left[\rho_{n}+r \sin (\eta / 2)\right]^{-1}
$$

In $D_{0}$, which we get by excluding from $D\left(s_{0}, \vartheta, \phi\right)$ the sequence of circles with centres at $-\lambda_{n}(n=1,2, \cdots)$ and radii $\epsilon, \epsilon$ being a small positive constant, we have evidently

$$
|K(s)|<K \text {, }
$$

where $K$ is a suitable constant. Since

$$
d_{n} \cdot r \cdot\left[\rho_{n+1}+r \sin (\eta / 2)\right]^{-1}=\operatorname{cosec}(\eta / 2)\left(d_{n}-d_{n+1}\right),
$$

taking account of (2.4) and (2.5), we have for any large $N$

$$
\begin{aligned}
\sum_{n=n_{1}}^{N}\left|c_{n}(s)-c_{n+1}(s)\right| & <K \operatorname{cosec}(\eta / 2) \sum_{n=n_{1}}^{N}\left(d_{n}-d_{n+1}\right) \\
& <K \operatorname{cosec}(\eta / 2) d_{n_{1}}
\end{aligned}
$$

uniformly in $D_{0}$.

Since $\sum_{n=1}^{\infty} b_{n}$ is convergent by the hypothesis, on account of (2.6) and du Bois-Reymond's Theorem [7, p. 315], $F(s)=\sum_{n=1}^{\infty} b_{n} c_{n}(s)$ is uniformly convergent in $D_{0}$. q.e.d.

LEMMA II. If (1.1) is absolutely convergent at $s=s_{0}$, then $\sum_{n=1}^{\infty}\left|a_{n}\right|\left|\left(\lambda_{1} \lambda_{2} \cdots \lambda_{n}\right)\left[\left(s+\lambda_{1}\right)\left(s+\lambda_{2}\right) \cdots\left(s+\lambda_{n}\right)\right]^{-1}\right|$ is uniformly convergent in the angular domain $D\left(s_{0}, \vartheta, \phi\right)$, where $D\left(s_{0}, \vartheta, \phi\right)$ has the same meaning as in Lemma I.

As a corollary, we get

LEMMA II'. If (1.1) with real $\lambda_{n}(n=1,2, \cdots)$ is absolutely convergent at $s=s_{0}$, then (1.1) is absolutely and uniformly convergent in the 
angular domain $D\left(s_{0}, \vartheta, 0\right)$.

Proof of Lemma II. Using the same notation as in Lemma I, (2.1) and (2.3) are also valid. Since

$$
\begin{aligned}
|| c_{n}(s)|-| c_{n+1}(s)|| & =\left|c_{n}(s)\right| \cdot|1-|\left(s_{0}+\lambda_{n+1}\right)\left(s+\lambda_{n+1}\right)^{-1}|| \\
& \leqq\left|c_{n}(s)\right| \cdot\left|\left(s-s_{0}\right)\left(s+\lambda_{n+1}\right)^{-1}\right|
\end{aligned}
$$

on account of (2.4) and (2.5), we obtain for any large $N$

$$
\sum_{n=n_{1}}^{N}|| c_{n}(s)|-| c_{n+1}(s)||<K \operatorname{cosec}(\eta / 2) \cdot d_{n_{1}}
$$

uniformly in $D_{0}$. Since $\sum_{n=1}^{\infty}\left|b_{n}\right|$ is convergent by the hypothesis, it results by virtue of (2.7) and du Bois-Reymond's theorem that $\sum_{n=1}^{\infty}\left|b_{n} \cdot c_{n}(s)\right|$ is uniformly convergent in $D_{0}$. q.e.d.

LEMMA III. If (1.1) is simply convergent at $s=s_{0}$, and furthermore there exists a point $s_{1}$ contained in the angular domain $D\left(s_{0}, \pi / 2-\phi\right)$ : $\left|\arg \left(s-s_{0}\right)\right| \leqq \pi / 2-\phi$, such that for a sufficiently large integer $n_{1}$, we have

$$
\left|\arg \left(s_{1}+\lambda_{n}\right)\right| \leqq \phi \quad \text { for } n \geqq n_{1},
$$

then (1.1) is uniformly convergent in the angular domain $D\left(s_{2}, \pi / 2-\phi\right)$, where $s_{2}=s_{1}+\epsilon \sec \phi, \epsilon$ being any small positive constant.

As an immediate consequence of Lemma III, we get

LEMMA III'. If (1.1) with real $\lambda_{n}(n=1,2, \cdots)$ is simply convergent at $s=s_{0}$, then (1.1) is uniformly convergent in the half-plane $D: \Re(s)$ $\geqq \Re\left(s_{0}\right)+\epsilon, \epsilon$ being any small positive constant.

In fact, we can put $\phi=0, s_{1}=\Re\left(s_{0}\right)$, and $s_{2}=\Re\left(s_{0}\right)+\epsilon$ in Lemma III. Proof of Lemma III. We first prove

$$
\left|s+\lambda_{n}\right| \geqq\left|s_{3}+\lambda_{n}\right|+\epsilon / 2 \quad \text { for } n \geqq n_{1},
$$

where $s \in D\left(s_{2}, \pi / 2-\phi\right)$, and $s_{3}=s_{1}+\epsilon / 2 \cdot \sec \phi$. In fact, putting $\alpha_{n}=\arg \left(s_{3}+\lambda_{n}\right)$, we have evidently

$$
\left|\alpha_{n}\right| \leqq \phi \quad \text { for } n \geqq n_{1} .
$$

Projecting the vector $\left(s+\lambda_{n}\right)$ perpendicularly on the vector $\left(s_{3}+\lambda_{n}\right)$, we get easily

$$
\left|s+\lambda_{n}\right| \geqq\left|s_{3}+\lambda_{n}\right|+\epsilon / 2 \cdot \sec \phi \cdot \cos \alpha_{n},
$$

so that, by (2.9),

$$
\left|s+\lambda_{n}\right| \geqq\left|s_{8}+\lambda_{n}\right|+\epsilon / 2 \text {, }
$$


which proves (2.8).

Let us put

$$
\begin{aligned}
b_{n}=a_{n}\left[\lambda_{1} \cdots \lambda_{n}\right]\left[\left(s_{3}+\lambda_{1}\right)\left(s_{3}+\lambda_{2}\right) \cdots\left(s_{3}+\lambda_{n}\right)\right]^{-1}, \\
c_{n}(s)=\left[\left(s_{3}+\lambda_{1}\right)\left(s_{3}+\lambda_{2}\right) \cdots\left(s_{3}+\lambda_{n}\right)\right] \\
\cdot\left[\left(s+\lambda_{1}\right)\left(s+\lambda_{2}\right) \cdots\left(s+\lambda_{n}\right)\right]^{-1}
\end{aligned}
$$

By (2.8) and arguments similar to those employed in the proof of Lemma I, we have

$$
\begin{aligned}
\left|c_{n}(s)-c_{n+1}(s)\right| & =\left|c_{n}(s)\right| \cdot\left|\left(s-s_{3}\right)\left(s+\lambda_{n+1}\right)^{-1}\right| \\
& <|K(s)| \cdot d_{n} \cdot\left(\rho_{n}+\epsilon / 2\right)^{-1}
\end{aligned}
$$

where

$$
\begin{aligned}
K(s) & =\left(s-s_{8}\right)\left[\left(s_{3}+\lambda_{1}\right) \cdots\left(s_{3}+\lambda_{n_{1}-1}\right)\right] \\
\cdot\left[\left(s+\lambda_{1}\right) \cdots\left(s+\lambda_{n_{1}-1}\right)\right]^{-1}, & \\
\rho_{n} & =\left|s_{8}+\lambda_{n}\right|, \quad d_{n}=\prod_{i=n_{1}}^{n} \rho_{i}\left(\rho_{i}+\epsilon / 2\right)^{-1} .
\end{aligned}
$$

Since $d_{n}\left(\rho_{n}+\epsilon / 2\right)^{-1}=2 / \epsilon \cdot\left(d_{n}-d_{n+1}\right)$, and $K(s)=O(1)$ in the domain $D_{0}$, as is easily seen by excluding from $D\left(s_{2}, \pi / 2-\phi\right)$ the sequence of small circles with centres at $-\lambda_{n}(n=1,2, \cdots)$ and radii $\epsilon^{\prime}>0$, by virtue of (2.11) we have

$$
\left|c_{n}(s)-c_{n+1}(s)\right|<2 K / \epsilon \cdot\left(d_{n}-d_{n+1}\right) \quad \text { for } n \geqq n_{1},
$$

uniformly in $D_{0}$, where $K$ is a suitable constant. Hence

$$
\sum_{n=n_{1}}^{N}\left|c_{n}(s)-c_{n+1}(s)\right|<2 K / \epsilon \cdot\left(d_{n_{1}}-d_{N+1}\right)<2 K / \epsilon \cdot d_{n_{1}}
$$

uniformly in $D_{0}$ for any given $N$.

Since (1.1) is simply convergent at $s=s_{0}$ by virtue of Lemma I, it follows from (2.12) and du Bois-Reymond's theorem that $F(s)$

$=\sum_{n-1}^{\infty} b_{n} c_{n}(s)$ is uniformly convergent in $D_{0}$. q.e.d.

Now we are in a position to prove Theorem $I$.

Proof of Theorem I. If (1.1) is simply (absolutely) convergent at $s=s_{0}$, then (1.1) is also simply (absolutely) convergent at $s=s_{1}$ with $\Re\left(s_{0}\right)<\Re\left(s_{1}\right)$ by virtue of Lemma I' (Lemma $\left.I^{\prime \prime}\right)$. Hence there exists a simple (absolute) convergence-abscissa $\sigma_{\diamond}\left(\sigma_{a}\right)$ of $(1.1)$, and we have evidently $\sigma_{\imath} \leqq \sigma_{a}$.

For any given $\epsilon>0,(1.1)$ is simply convergent at $s=\sigma_{s}+\epsilon / 2$, so that by Lemma $\mathrm{III}^{\prime},(1.1)$ is uniformly convergent for $\Re(s) \geqq \sigma_{\imath}+\epsilon$. But since (1.1) is not simply convergent on $s=\sigma_{8}-\epsilon,(1.1)$ is not uni- 
formly convergent for $\Re(s) \geqq \sigma_{\varepsilon}-\epsilon$. Hence $\sigma_{u}$ coincides with $\sigma_{\imath}$. Thus we have $\sigma_{s}=\sigma_{u} \leqq \sigma_{a}$. q.e.d.

3. Proof of Theorem II. Since $\sum_{n=1}^{\infty} 1 / r_{n}<+\infty$, the infinite product $g(s)=\prod_{n=1}^{\infty}\left(1+s / \lambda_{n}\right)$ converges, so that we have

$$
0<|g(s)|<+\infty \quad \text { for } s \neq-\lambda_{n}(n=1,2, \cdots) .
$$

Let us put

$$
c_{n}(s)=\left[\lambda_{1} \cdots \lambda_{n}\right]\left[\left(s+\lambda_{1}\right)\left(s+\lambda_{2}\right) \cdots\left(s+\lambda_{n}\right) \cdot\right]^{-1}=\left[g_{n}(s)\right]^{-1},
$$

where $g_{n}(s)=\prod_{i=1}^{n}\left(1+s / \lambda_{i}\right)$. Since

$$
c_{n}(s)-c_{n+1}(s)=\left[g_{n}(s) \cdot \lambda_{n+1}\right]^{-1} \cdot s\left(1+s / \lambda_{n+1}\right)^{-1},
$$

by (3.1) we get

$$
\left|c_{n}(s)-c_{n+1}(s)\right|<K_{1}|g(s)|^{-1} \cdot 1 / r_{n+1} \quad \text { for } n \geqq n_{1},
$$

where (i) $K_{1}$ is a suitable constant, (ii) $n_{1}$ is a sufficiently large integer. Hence

$$
\sum_{n=n_{1}}^{\infty}\left|c_{n}(s)-c_{n+1}(s)\right|<K_{1}|g(s)|^{-1} \cdot \sum_{n=n_{1}}^{\infty} 1 / r_{n+1}<+\infty .
$$

If $\sum_{n=1}^{\infty} a_{n}$ converges, then by (3.2) and du Bois-Reymond's theorem, $F(s)=\sum_{n=1}^{\infty} a_{n} c_{n}(s)$ also converges for $s$ different from $-\lambda_{n}$ $(n=1,2, \cdots)$.

Next suppose that $F\left(s_{0}\right)=\sum_{n=1}^{\infty} b_{n}\left(s_{0}\right)$ converges for $s=s_{0} \neq-\lambda_{n}$ $(n=1,2, \cdots)$, where

$$
b_{n}\left(s_{0}\right)=a_{n}\left[\lambda_{1} \cdots \lambda_{n}\right]\left[\left(s_{0}+\lambda_{1}\right)\left(s_{0}+\lambda_{2}\right) \cdots\left(s_{0}+\lambda_{n}\right)\right]^{-1} .
$$

Since $g_{n+1}\left(s_{0}\right)-g_{n}\left(s_{0}\right)=g_{n}\left(s_{0}\right) \cdot s_{0} / \lambda_{n+1}$, by (3.1) we get

$$
\left|g_{n+1}\left(s_{0}\right)-g_{n}\left(s_{0}\right)\right|<\left|g\left(s_{0}\right)\right| \cdot K_{2} / r_{n+1} \quad \text { for } n \geqq n_{2} \text {, }
$$

where (i) $K_{2}$ is a suitable constant, (ii) $n_{2}$ is a sufficiently large integer, so that

$$
\sum_{n=n_{2}}^{\infty}\left|g_{n+1}\left(s_{0}\right)-g_{n}\left(s_{0}\right)\right|<\left|g\left(s_{0}\right)\right| \cdot K_{2} \cdot \sum_{n=n_{2}}^{\infty} 1 / r_{n+1}<+\infty .
$$

Since $\sum_{n=1}^{\infty} b_{n}\left(s_{0}\right)$ converges, by (3.3) and du Bois-Reymond's theorem, $\sum_{n=1}^{\infty} a_{n}=\sum_{n=1}^{\infty} b_{n}\left(s_{0}\right) g_{n}\left(s_{0}\right)$ is also convergent.

By entirely similar arguments, we can prove that the necessarysufficient condition for (1.1) to converge absolutely at $s=s_{0}$ different from $-\lambda_{n}(n=1,2, \cdots)$ is that $\sum_{n=1}^{\infty}\left|a_{n}\right|<+\infty$.

If $\sum_{n=1}^{\infty} 1 / r_{n}<+\infty$ and $\phi_{n}=0(n=1,2, \cdots)$, then the second part 
of Theorem II immediately follows from Theorem I and what is proved above.

4. Proof of Theorem III. Let us put

$$
k=\limsup _{n \rightarrow \infty} 1 / l_{n} \cdot \log \left|\sum_{n=1}^{n} a_{,} \exp \left(\phi\left(l_{v}\right)-\phi\left(l_{n}\right)\right)\right| .
$$

We shall first establish the inequality

$$
k \leqq \sigma_{8} .
$$

Since (1.1) is simply convergent for $s=\sigma>\sigma_{e}$, there exists a constant $K$ such that

$$
\left|S_{n}\right|<K \quad(n=1,2, \cdots),
$$

where

$$
S_{n}=\sum_{i=1}^{n} a_{i}\left[\lambda_{1} \cdots \lambda_{i}\right]\left[\left(\sigma+\lambda_{1}\right)\left(\sigma+\lambda_{2}\right) \cdots\left(\sigma+\lambda_{i}\right)\right]^{-1} .
$$

Putting $S_{0}=0$ and applying Abel's transformation, we have

$$
\sum_{i=1}^{n} a_{i} \exp \left(\phi\left(l_{i}\right)\right)=\sum_{i=1}^{n-1} S_{i}(f(i)-f(i+1))+S_{n} f(n),
$$

where $f(i)=\exp \left(\phi\left(l_{i}\right)\right) \cdot \prod_{k=1}^{i}\left(1+\sigma / \lambda_{h}\right)$. On the other hand,

$$
f(i)=Q(\sigma) \cdot \exp \left\{\phi\left(l_{i}\right)+l_{i}\left(\sigma+\epsilon_{i}(\sigma)\right)\right\}
$$

for $i>n_{1}$,

where

$$
\begin{aligned}
& Q(\sigma)=\prod_{n=1}^{n_{1}}\left(1+\sigma / \lambda_{n}\right) \exp \left(-\sigma / \lambda_{n}\right), \\
& \lim _{i \rightarrow \infty} \epsilon_{i}(\sigma)=0,
\end{aligned}
$$

$$
n_{1} \text { is a sufficiently large integer. }
$$

In fact, since

$$
(1+x)=\exp \left(x+x^{2} \cdot \rho(x)\right),|\rho(x)| \leqq 1 \quad \text { for }|x| \leqq 1 / 2,
$$

we can easily obtain the relation

$$
\begin{aligned}
f(i) & =\prod_{n=1}^{n_{1}}\left(1+\sigma / \lambda_{n}\right) \exp \left(-\sigma / \lambda_{n}\right) \\
& \times \exp \left\{\phi\left(l_{i}\right)+\sigma l_{i}+\sigma^{2} \cdot \vartheta(\sigma)\left(\sum_{n=1}^{i} 1 / \lambda_{n}^{2}\right)\right\},
\end{aligned}
$$


where (i) $\left|\sigma / \lambda_{n}\right| \leqq 1 / 2$ for $n>n_{1}$, (ii) $|\vartheta(\sigma)| \leqq 1$. Since $\lim _{i \rightarrow \infty} 1 / l_{i}$ $\cdot \sum_{n=1}^{i} 1 / \lambda_{n}^{2}=0,(4.6)$ gives (4.5).

Taking account of the hypothesis (d) part (i), we can easily prove that

$$
g(i) \uparrow \infty \quad \text { for } i>n_{2},
$$

where

(i) $g(i)=\exp \left(\phi\left(l_{i}\right)+l_{i}\left(\sigma+\epsilon_{i}(\sigma)\right)\right)$,

(ii) $n_{2}$ is a sufficiently large integer.

Therefore, putting $N=\operatorname{Max}\left(n_{1}, n_{2}\right)$, by (4.4) and (4.3) we have

$$
\begin{aligned}
\left|\sum_{i=1}^{n} a_{i} \exp \left(\phi\left(l_{i}\right)\right)\right| \leqq K & \cdot\left|\sum_{i=1}^{N} f(i)-f(i+1)\right|+K|Q(\sigma)| \\
& \cdot\left\{\sum_{i=N+1}^{n-1} g(i+1)-g(i)+g(n)\right\},
\end{aligned}
$$

so that for sufficiently large $n$,

$$
\left|\sum_{i=1}^{n} a_{i} \exp \left(\phi\left(l_{i}\right)\right)\right|<3 K \cdot|Q(\sigma)| \cdot g(n) .
$$

Hence $k \leqq \sigma+\lim _{n \rightarrow \infty} \epsilon_{n}(\sigma)=\sigma$. Letting $\sigma \rightarrow \sigma_{\text {, }}$, we have $k \leqq \sigma_{\text {, }}$, which proves (4.2).

Next we shall prove

$$
\sigma_{\iota} \leqq k \text {. }
$$

By the definition of $k$, for any given $\delta>0$, there exists a constant $N$ such that

$$
\left|T_{n}\right|<U_{n}=\exp \left\{\phi\left(l_{n}\right)+l_{n}(k+\delta / 2)\right\} \quad \text { for } n \geqq N,
$$

where $T_{n}=\sum_{k=1}^{n} a_{i} \exp \left(\phi\left(l_{i}\right)\right)$. Taking account of $a_{n}=\left(T_{n}-T_{n-1}\right)$ $\exp \left(-\phi\left(l_{n}\right)\right)$, by Abel's transformation we get

$$
\begin{gathered}
\sum_{i=N+1}^{M} a_{i}\left[\lambda_{1} \cdots \lambda_{i}\right]\left[\left(k+\delta+\lambda_{1}\right)\left(k+\delta+\lambda_{2}\right) \cdots\left(k+\delta+\lambda_{i}\right)\right]^{-1} \\
=\sum_{i=N+1}^{M-1} T_{i}(h(i)-h(i+1))-T_{N} h(N+1)+T_{M} h(M)
\end{gathered}
$$

where $h(i)=\exp \left(-\phi\left(l_{i}\right)\right) \cdot\left[\prod_{k-1}^{k}\left(1+(k+\delta) / \lambda_{k}\right)\right]^{-1}$. By arguments similar to those employed before we may write

$$
h(i)=K \cdot g(i) \quad \text { for } i>n_{1},
$$

where 


$$
K=\left[\prod_{n=1}^{n_{1}}\left(1+(k+\delta) / \lambda_{n}\right) \cdot \exp \left(-(k+\delta) / \lambda_{n}\right)\right]^{-1}
$$

(ii) $g(i)=\exp \left\{-\left(\phi\left(l_{i}\right)+l_{i}\left(k+\delta+\epsilon_{i}\right)\right)\right\}$,

(iii) $\lim _{i \rightarrow \infty} \epsilon_{i}=0$,

(iv) $\quad n_{1}$ is a sufficiently large integer.

Accordingly, by (4.8), (4.9), and (4.10) we obtain

$$
\begin{aligned}
& \left|\sum_{i=N+1}^{M} a_{i}\left[\lambda_{1} \cdots \lambda_{i}\right]\left[\left(k+\delta+\lambda_{1}\right)\left(k+\delta+\lambda_{2}\right) \cdots\left(k+\delta+\lambda_{i}\right)\right]^{-1}\right| \\
& \leqq|K|\left\{\sum_{i=N+1}^{M-1} U_{i}|g(i)-g(i+1)|+U_{N} g(N+1)+U_{M g} g(M)\right\} .
\end{aligned}
$$

On the other hand, for sufficiently large $i$, we get easily

$$
\begin{aligned}
|g(i)-g(i+1)| & =O\left(\left|\int_{l_{i}}^{l_{i+1}} \frac{d}{d x} \exp (-(\phi(x)+x(k+\delta))) d x\right|\right) \\
& =O\left(1 / U_{i} \cdot \int_{l_{i}}^{l_{i+1}} \exp (-\delta / 2 \cdot x)\left|\phi^{\prime}(x)\right| d x\right) .
\end{aligned}
$$

Hence, by (4.9), (4.10) and the hypothesis (d) part (ii), we get for sufficiently large $N$

$$
\begin{aligned}
& \left|\sum_{i=N+1}^{M} a_{i}\left[\lambda_{1} \cdots \lambda_{i}\right]\left[\left(k+\delta+\lambda_{1}\right)\left(k+\delta+\lambda_{2}\right) \cdots\left(k+\delta+\lambda_{i}\right)\right]^{-1}\right| \\
& =O\left(\int_{l_{M+1}}^{l_{M}} \exp (-\delta / 2 \cdot x)\left|\phi^{\prime}(x)\right| d x\right)+O\left(\exp \left(-l_{N+1}\left(\delta / 2+\epsilon_{N+1}\right)\right)\right) \\
& \quad+O\left(\exp \left(-l_{M}\left(\delta / 2+\epsilon_{M}\right)\right)\right)=o(1),
\end{aligned}
$$

so that (1.1) is simply convergent at $s=k+\delta$. Therefore

$$
\sigma_{.}<k+\delta
$$

for any given $\delta>0$, which proves (4.6).

Thus, by (4.3), (4.7), and Theorem 1, we have

$$
k=\sigma_{\iota}=\sigma_{u},
$$

which proves (a) of Theorem III. By the slight modification of the above arguments, we can also prove (b) of Theorem III.

5. Proof of corollaries. By M. Fujiwara's theorem [8], the simple convergence-abscissa $\sigma_{\bullet}(G)$ and the absolute convergence-abscissa 
$\sigma_{a}(G)$ of $G(s)$ are given respectively by

$$
\begin{aligned}
& \sigma_{s}(G)=\limsup _{n \rightarrow \infty} 1 / l_{n} \cdot \log \left|\sum_{n=1}^{n} a_{\nu} \exp \left(l_{n}^{2}-l_{n}^{2}\right)\right|, \\
& \sigma_{a}(G)=\limsup _{n \rightarrow \infty} 1 / l_{n} \cdot \log \left\{\sum_{n=1}^{n}\left|a_{\nu}\right| \exp \left(l_{n}^{2}-l_{n}^{2}\right)\right\} .
\end{aligned}
$$

Since $\phi(x)=x^{2}$ evidently satisfies the conditions of Theorem III, taking account of Theorem III and (5.1) we get

$$
\sigma_{a}=\sigma_{s}(G), \quad \sigma_{a}=\sigma_{a}(G),
$$

which proves Corollary I.

By T. Kojima's theorem [9], we may write

$$
\begin{aligned}
& \sigma_{\triangleleft}(G)=\limsup _{x \rightarrow \infty} 1 / x \cdot \log \left|\sum_{[x] \leqq l_{n}<x} a_{n}\right|, \\
& \sigma_{a}(G)=\limsup _{x \rightarrow \infty} 1 / x \cdot \log \left\{\sum_{[x] \leqq l_{n}<x}\left|a_{n}\right|\right\},
\end{aligned}
$$

so that the first part of Corollary II follows immediately from Corollary I. On the other hand, by a well known theorem $[10$, p. 49], we have

$$
0 \leqq \sigma_{a}(G)-\sigma_{s}(G) \leqq \limsup _{n \rightarrow \infty} 1 / 1_{n} \cdot \log n,
$$

which proves the second part of corollary II.

\section{REFERENCES}

1. E. Landau, Über die Grundlagen der Theorie der Fakultatenreihen, Münchener Ber. vol. 36 (1906).

2. S. Pincherle, Sulla serie fattoriale generalizzate, Rend. Circ. Mat. Palermo vol. 37 (1914).

3. G. Belardinelli, Su alcune serie de funzioni rationali, Rend. Circ. Mat. Palermo vol. 47 (1923).

4. T. Fort, Generalized factorial series, Trans. Amer. Math. Soc. vol. 31 (1929).

5. - The general theory of factorial series, Bull. Amer. Math. Soc. vol. 36 (1930).

6. N. E. Nörlund, Lę̧ons sur les serries d'interpolation, Paris, 1926.

7. K. Knopp, Theory and application of infinite series, London and Glasgow, 1928.

8. M. Fujiwara, On the convergence-abscissa of general Dirichlet's series, Tôhoku Math. J. vol. 6 (1914).

9. T. Kojima, On the convergence-abscissa of general Dirichlet's series, Tohoku Math. J. vol. 6 (1914).

10. D. V. Widder, The Laplace transform, Princeton, 1946.

WASEDA UnIVERSity, Toxyo 